\title{
Investigation of coupling effect between a unidirectional air waveguide and two cavities with one-way rotating state
}

\author{
Yongfeng Gao ${ }^{1 *}$, Liu He ${ }^{1}$, Zhen Jiang ${ }^{1}$, Jun Zhou ${ }^{2}$, Yuejia Shi ${ }^{1}$, Wenfang Bai ${ }^{1}$ \\ ${ }^{1}$ School of Mechanical Engineering, Jiangsu University, Zhenjiang Jiangsu 212013, China \\ ${ }^{2}$ Department of Microelectronic Science and Engineering, School of Physical Science and Technology, \\ Ningbo University, Ningbo Zhejiang 315211, China
}

\begin{abstract}
In the paper we present an implementation of resonance coupling between a unidirectional air waveguide and two cavities with one-way rotating state in a two-dimensional dielectric photonic crystal and a magneto-optical photonic crystal. We theoretically calculate the dispersion curves of the line defect waveguide and the dispersion curves of waveguide-cavity interaction, respectively. Numerical simulations show that, via waveguide-cavity coupling, a series of interesting resonance phenomena can be generated in the system. By modifying direction of the applied external magnetic field, storing and discharging of photonic energy can be realized. Our work may provide a practical and useful guidance for optical communications.
\end{abstract}

Keywords: waveguide-cavity coupling, magneto-optical photonic crystal, unidirectional air waveguide, one-way rotating state, finite element method.

\section{Introduction}

Photonic crystals (PCs) is a kind of artificial microstructure which has been widely used in trapping and guiding of electromagnetic (EM) wave in many fields because of their unique characteristics, such as photonic band gaps (PBGs) and photonic location $[1,2]$. The theoretical basis of PCs band system was analogous to electronic band structures $[\underline{3}, \underline{4}]$. RAGHU and HALDANE $[\underline{5}, \underline{6}]$ predicted the existence of one-way edge modes (OWEM) of electromagnetic waves with magneto-optical photonic crystals (MOPCs) and further extended the application in the quantum Hall effect. WANG et al. demonstrated and observed the OWEM in the microwave regime in the experiment with regular PCs and MOPCs $[\underline{7}, \underline{8}]$. Hence, OWEM has provided a possible way for realizing new photonic devices and opened a frontier gate to condensed matter physics.

Based on OWEM of MOPCs, various optical nonreciprocity devices have been proposed in optical integration circuits, such as optical waveguide isolators $[\underline{9}, \underline{10}]$, circulators [11], delay waveguides [12], unidirectional filters [13], etc. However, these devices often operate with low efficiencies, and are easily influenced by a light leak 
in the interface between regular PCs and MOPCs. Researchers have proposed waveguide-cavity coupling schemes [14-16], which can enhance optical device performances and realize flexible tunability of the system. Recently, FANG et al. [17-19] designed a unidirectional air waveguide with single-mode transmission in MOPCs based on the waveguide coupling theory [20]. In this work, we design a configuration consisting of unidirectional air waveguide and two cavities with a one-way rotating state. We investigate the coupling resonance frequencies between PCs cavities with a one-way rotating state and a unidirectional air waveguide, which reveal a series of related interesting phenomena of waveguide-cavity resonance. Furthermore, propagation direction of one-way electromagnetic modes depends on the direction of applied external dc magnetic field. We employ the model to achieve two waveguide-coupled ring resonators with the capability of storage and discharge of light by changing the initial direction of the applied external magnetic field.

\section{Model and method}

Here we propose a PCs structure composed of a line waveguide and two same cavities, as shown in Fig. 1, where the two cavities are constructed by the alumina $\left(\mathrm{Al}_{2} \mathrm{O}_{3}\right)$ (in gray) cylindrical rods in a square lattice array. The outer-layered frame is constructed by yttrium-iron-garnet (YIG) (in blue) cylindrical rods in a square lattice array tilted by

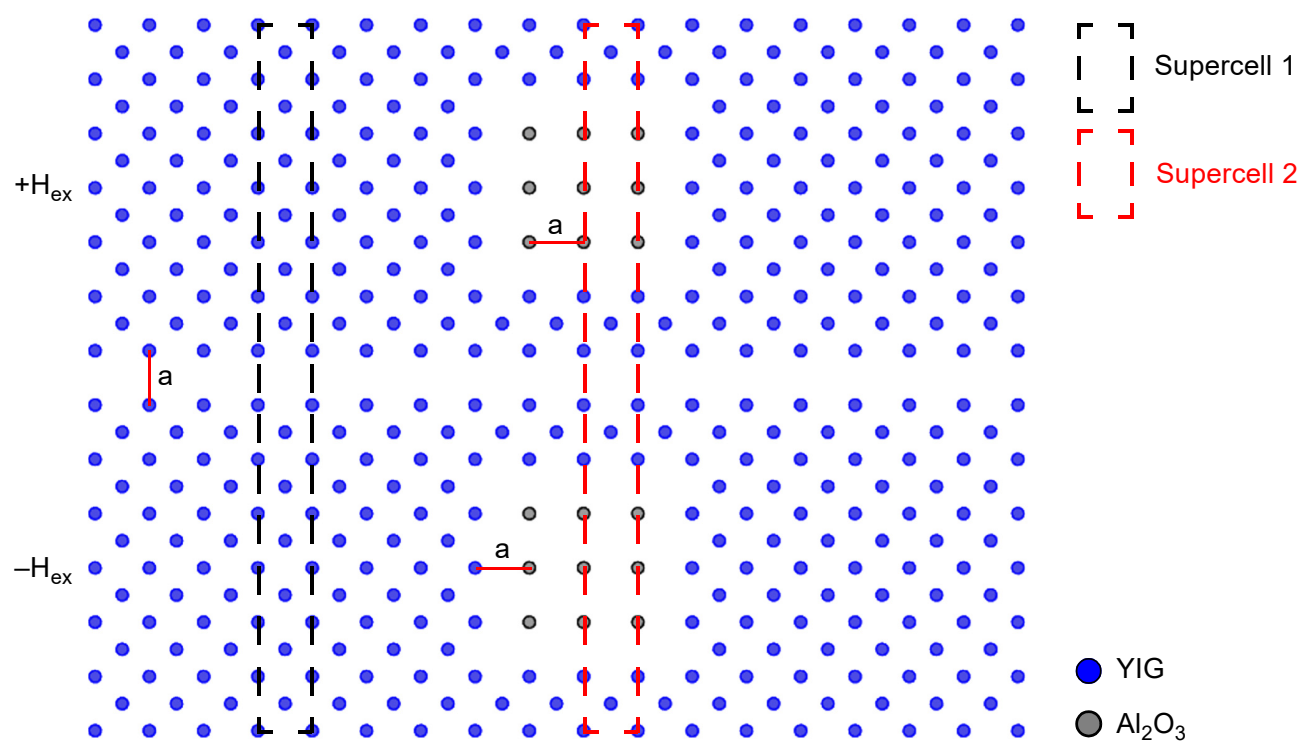

Fig. 1. Schematic of 2D PCs modeling. The upper and lower parts of the line defect waveguide are the total applied external dc magnetic fields along $+z$-axis, $-z$-axis, respectively; supercell $1-$ the supercell of a unidirectional air waveguide to calculate the PBGs, and supercell 2 - the supercell coupling between a unidirectional air waveguide and two cavities. 


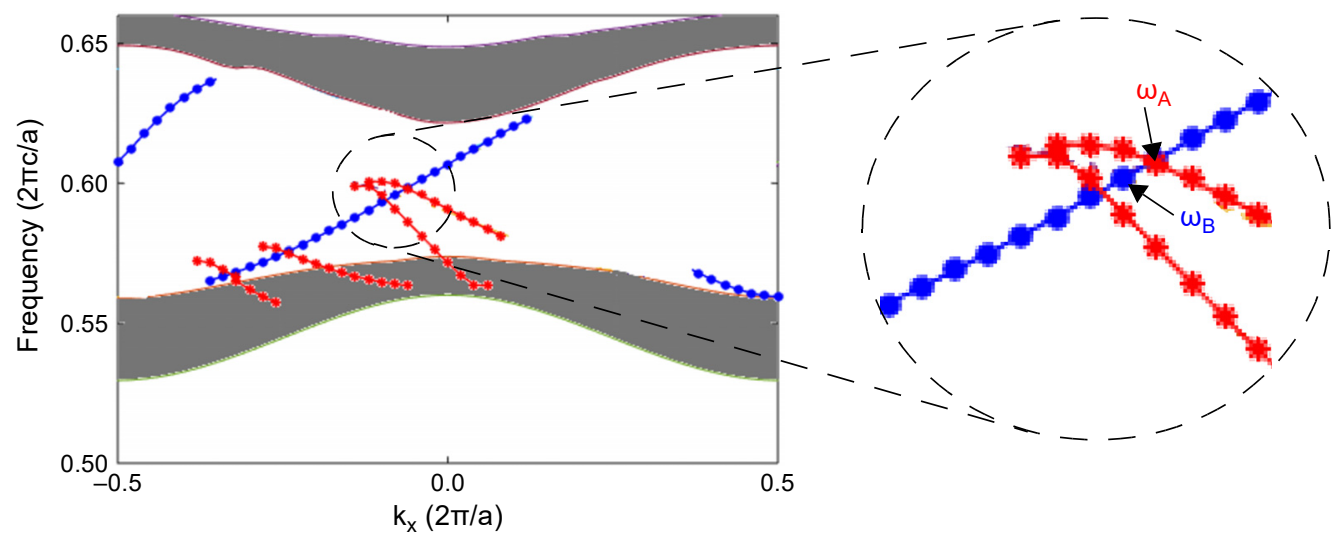

Fig. 2. The dispersion curves of the coupled modes; the three blue dot-lines denote dispersion curves of a line defect waveguide, the four red dot-lines denote dispersion curves of coupling between a unidirectional air waveguide and two cavities, where the crossed points denote resonance frequencies of waveguide-cavity interaction; enlarged view: $\omega_{\mathrm{A}}$ denotes corresponding frequency of the crossed point between blue and red lines, namely, which is the maximum resonance frequency, $\omega_{\mathrm{B}}$ denotes the non-resonance frequency.

45 degrees with respect to regular PCs, i.e. the lattice constant turns into $a / \sqrt{2}$, where $a=500 \mathrm{~nm}$ and cylindrical rods radii $r=0.11 a$. The material parameters in the paper refer to these references [21-24]. For simplicity, based on the Maxwell equations [25], we calculate the configuration bands and eigenvalue frequencies, and numerically simulate dispersion relations of eigenmodes with the finite element method (FEM) using COMSOL Multiphysics. The projected band dispersion curves of the line defect waveguide and the dispersion curves of waveguide-cavity interaction are obtained through calculating the supercell 1 and supercell 2 [26-30], respectively.

As is shown in Fig. 2, the three blue curves represent coupling dispersion curves of the unidirectional air waveguide, which show that the group velocity $\left(v_{\mathrm{g}}=\mathrm{d} w / \mathrm{d} k_{x}\right)$ of the unidirectional air waveguide points to direction according to the tonelessly slope of curves. The red four curves represent the dispersion curves of waveguide-cavity coupling, indicating that EM wave is a one-way rotating state in the cavities and their rotating direction depends on the direction of the applied external dc magnetic field. In addition, the middle blue curves and red ones are crossing at four points, implying that there are four resonance frequencies for this structure. Therefore, four operation channels to meet a unidirectional air waveguide and two cavities simultaneously can be operated at four resonance frequencies, respectively.

\section{Result and discussion}

As discussed above, we choose one of resonance frequencies and demonstrate the resonance phenomenon. In Fig. 3a, we get an $E_{z}$ field distribution program with the maximum 
resonance frequency $\omega_{\mathrm{A}}=0.5939(2 \pi c / a)$. It is also observed that the unidirectional air waveguide with single-mode transmission is stable and the two cavities modes are symmetric with regard to the horizontal center line (black dashed line) because the struc-
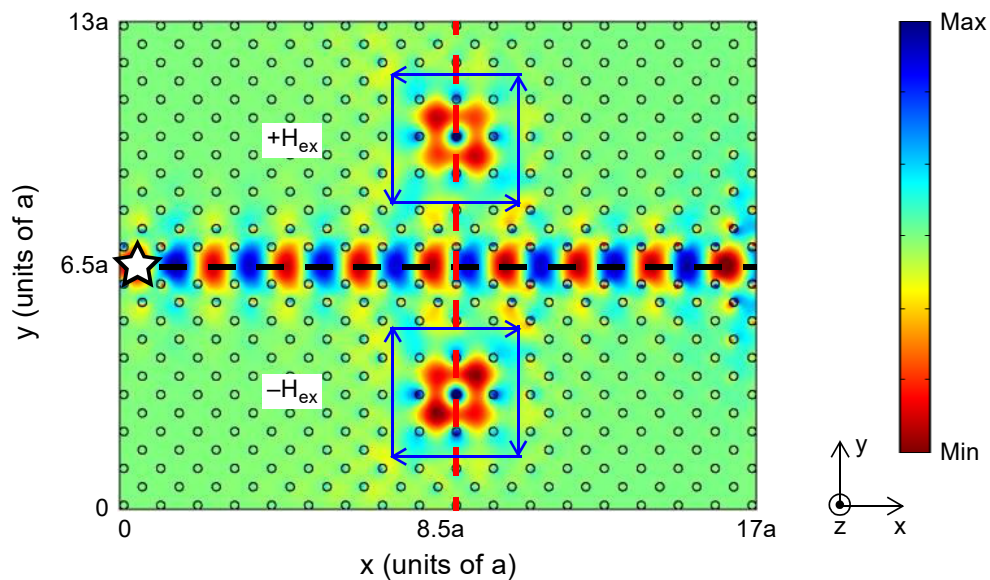

a

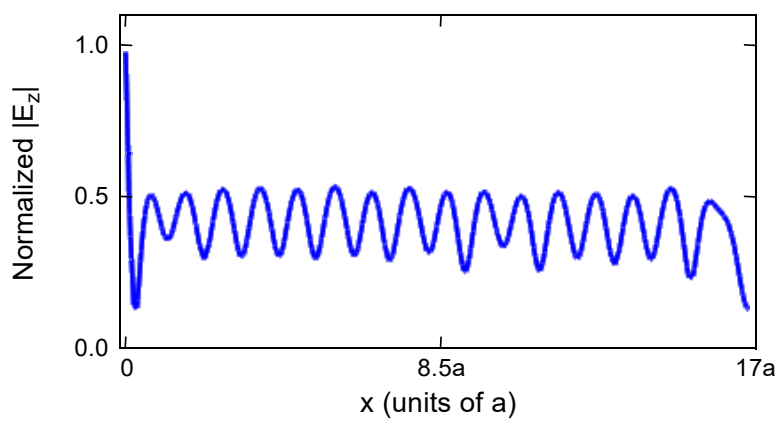

b

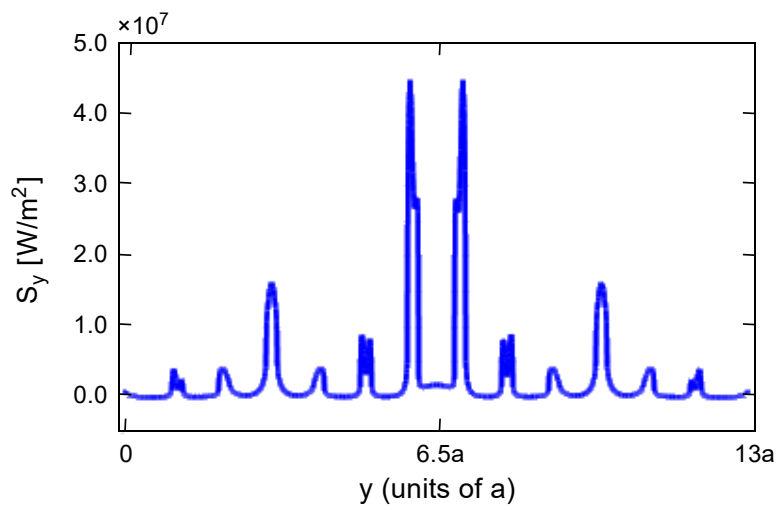

C

Fig. 3. Field distribution $\left(E_{z}\right)$ of waveguide-cavity interaction with resonance frequency $\omega_{\mathrm{A}}=0.5939(2 \pi c / a)$; a star denotes a line polarized source, blue arrows denote rotating direction of cavities with one-way modes, the black dashed line denotes the horizontal line at the waveguide center, the red dashed line denotes the vertical line at the structure center (a). The normalized $\left|E_{Z}\right|$ along the horizontal black dashed line (b). The time-average energy flow along the vertical red dashed line (c). 


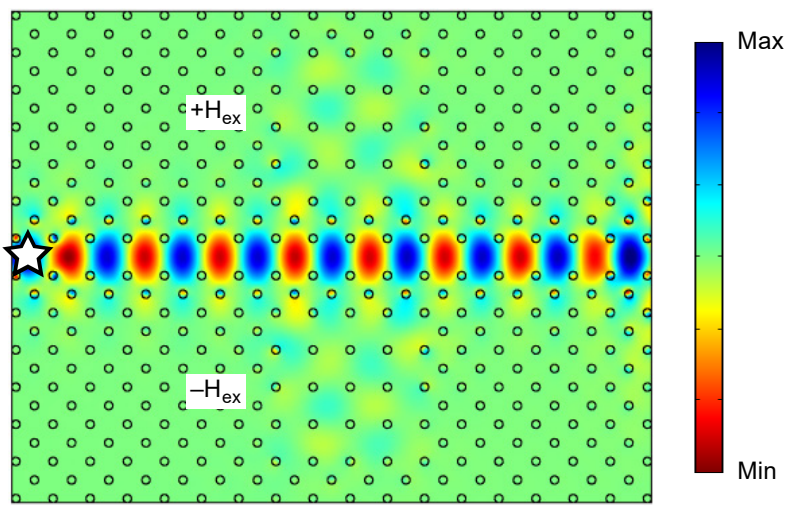

Fig. 4. Field distribution $\left(E_{z}\right)$ with non-resonance frequency $\omega_{\mathrm{B}}=0.5919(2 \pi c / a)$.

ture is symmetric. Noteworthily, the mode in the upper cavity is anti-clockwise rotational and the lower one is clockwise rotational because of the external magnetic field applied in opposite directions for the upper and lower parts, demonstrating that EM wave is a one-way rotating state in the cavities. We employ $2 \mathrm{D}$ cut line to detect the energy distribution. In Fig. 3b, normalized $E_{z}$ field distribution of the unidirectional air waveguide forms a nearly zero-loss sine wave along the $x$-direction, indicating that the energy loss of the unidirectional air waveguide is vastly slight. In Fig. 3c, we plot the time-average energy flows in the $y$-direction along the vertical red dashed line, which reveals that a lot of energy is accumulated in the unidirectional air waveguide and the other distributes in the cavity. We give a non-resonant frequency $\omega_{\mathrm{B}}=0.5919(2 \pi c / a)$, and get alone working unidirectional air waveguide transmission modes with sign-mode. However, the two cavities are not working. Consequently, the two cavities are not motivated and are not resonance modes, as shown in Fig. 4. For this case, we can realize different functions with different excited frequencies, e.g., it can be used to construct an isolator based on the one-way transmission with non-resonant frequency, and made a multi-channels waveguide-cavity ring resonator with the resonance frequencies.

In order to investigate the relation between coupling resonance of two cavities with a one-way rotating state and the line defect waveguide, we choose the maximum resonance frequency as an excited resonant source. We find that the lower cavity forms a clockwise rotating state and the upper cavity forms an anti-clockwise rotating state; nonetheless, $E_{z}$ field distribution intensity of the upper cavity is weaker than that of the lower cavity because of the system energy loss. As shown in Fig. 5a, waveguide coupling induces OWEM in the line defect waveguide. This phenomenon can be explained by the following two reasons. Firstly, electric field patterns of anti-clockwise rotation in the upper cavity and clockwise rotation in the lower cavity have been constantly accumulated and strengthened for leaking light crossing the three rows YIG rods, which produces light energy convergence in the line defect waveguide. EM wave propagating along the $x$-direction is called forward transmission for the structure. Secondly, backward transmission of the EM wave is completely suppressed owing to the nonre- 

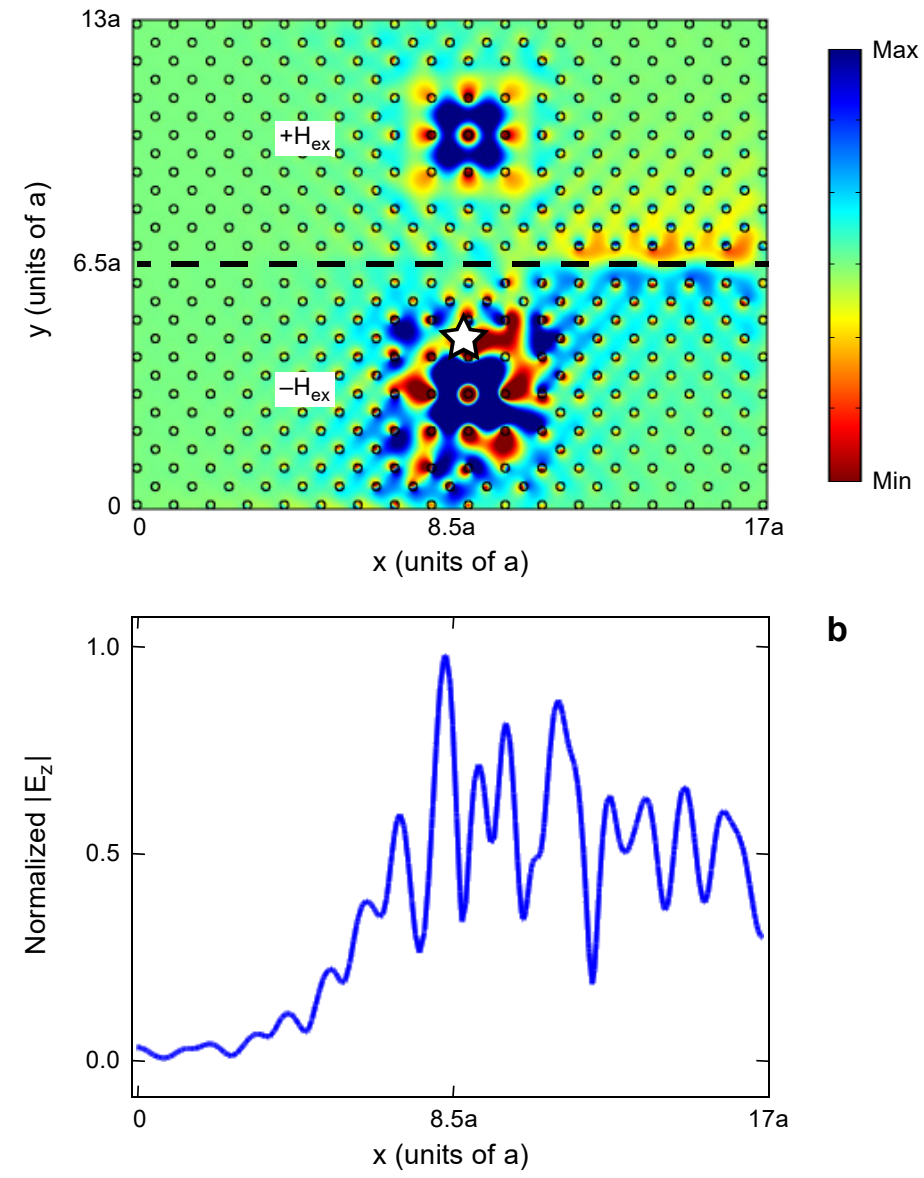

Fig. 5. The field distribution $\left(E_{z}\right)$ of coupling resonance between two cavities with resonance frequency $\omega_{\mathrm{A}}=0.5939(2 \pi c / a)(\mathbf{a})$. The normalized $\left|E_{z}\right|$ of waveguide excited by two cavities resonating along the horizontal black dashed line (b).

ciprocal waveguide feature. To test the OWEM, we detect the normalized $E_{z}$ field distribution along the $x$-direction. It is shown that at the waveguide distance from $x=8.5 a$ to $x=0$, the normalized $\left|E_{z}\right|$ is quickly decreased to zero, and at the range from $x=8.5 a$ to $x=17 a$, the normalized $\left|E_{z}\right|$ value is about 0.5 (see Fig. $5 \mathbf{b}$ ). The result shows that OWEM is induced in the line defect waveguide from $x=8.5 a$ to $x=17 a$. Hence, the resonance phenomena between two cavities and the line defect waveguide are in line with the analysis.

To our knowledge, propagation direction of the one-way electromagnetic modes depends on the direction of external dc magnetic field applied. The unidirectional air waveguide along the $x$-direction is partly blocked by applying the external magnetic field zone and direction as shown in Fig. 6. Eventually, we obtain two ring resonators with the capacity of storage. To see what happens in the system, we check the real-space electric energy distribution of the Poynting vector $\mathbf{S}=\operatorname{Re}\left[\mathbf{E} \times \mathbf{H}^{*}\right) / 2$ ) [25], which de- 


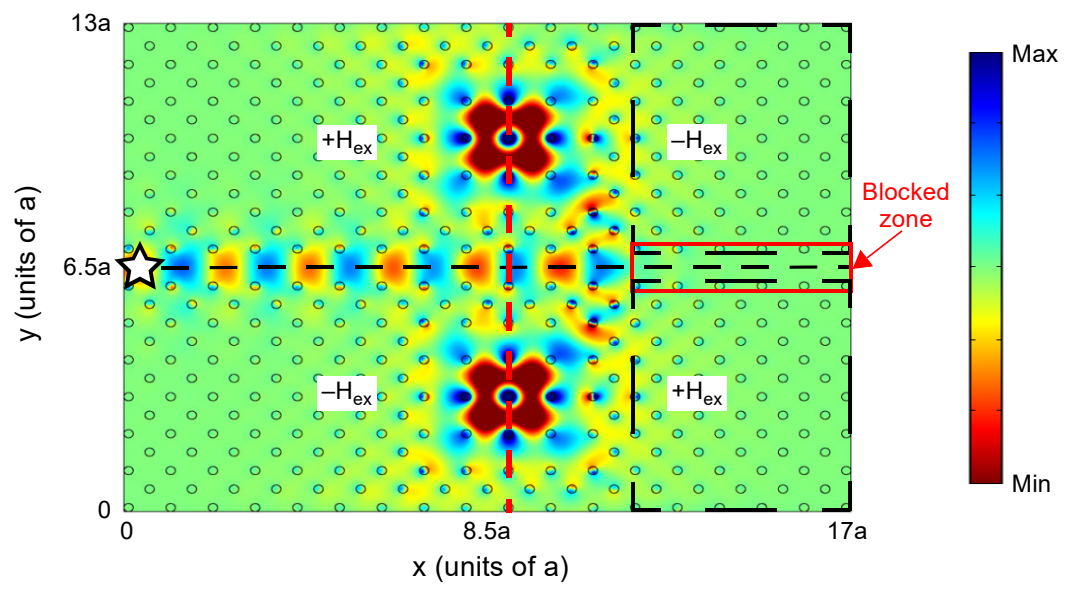

Fig. 6. The field distribution $\left(E_{z}\right)$ of two ring resonators. The black dashed rectangles denote the area of magnetic field applied.

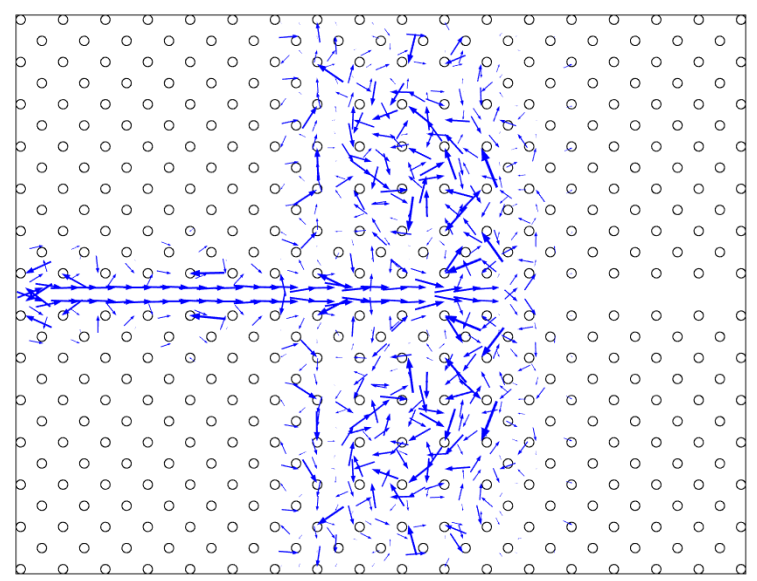

Fig. 7. Poynting vector corresponding to Fig. 6.

scribes the energy flow in the present EM system (Fig. 7). The direction of energy flow is depicted in blue arrows, which shows that the line defect waveguide is one-way transport until it is suppressed at the blocked zone. From Fig. 8a, the normalized $E_{z}$ field intensity for forward transmission in the line defect waveguide is much stronger than that for the case shown in Fig. $3 \mathbf{b}$. The reason is that photonic energy is constantly accumulated because the forward transmission of a unidirectional air waveguide is blocked. Consequently, photonic energy of two resonance cavities is also increasing. We plot the time-average energy flow along the vertical red dashed line (Fig. 8b), which reveals that photonic energy of the resonance cavity is much stronger than that shown in Fig. 3c. Here two ring resonators with high-capacity can be achieved. In addition, according to the resonators feature, it can be used in the laser cavity to realize the light amplification or modulation of the quality factor $(Q$ factor) [21] . 


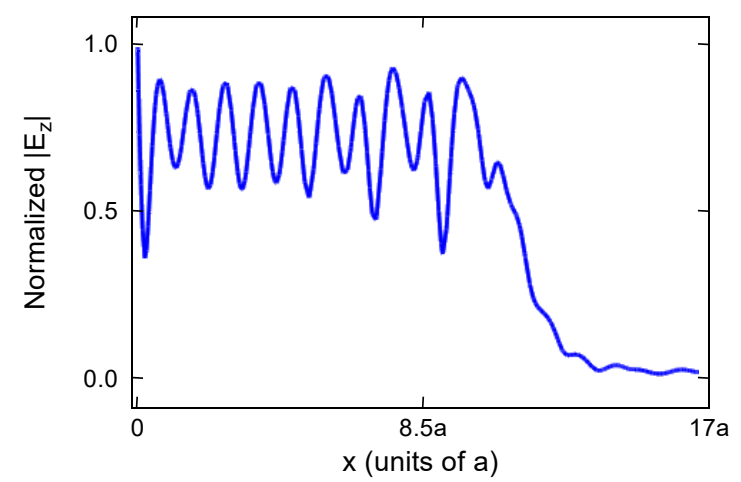

a

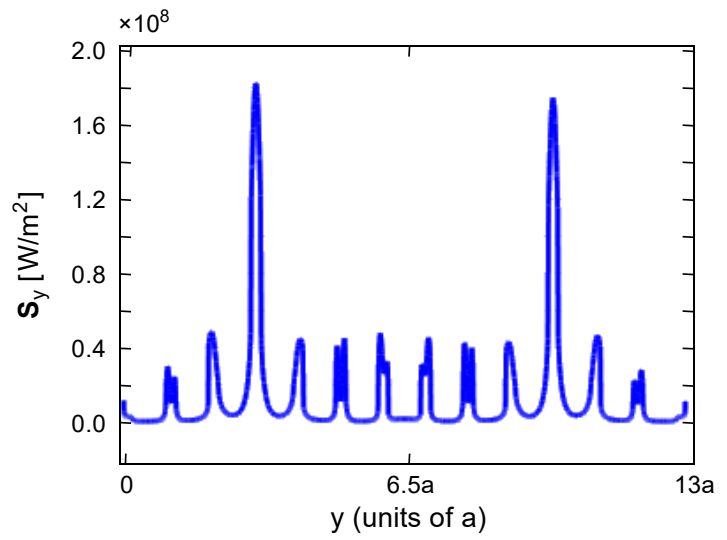

b

Fig. 8. The normalized $\left|E_{z}\right|$ along the horizontal black dashed line (see Fig. 6) (a). The time-average energy flow along the red vertical dashed line (see Fig 6) (b).

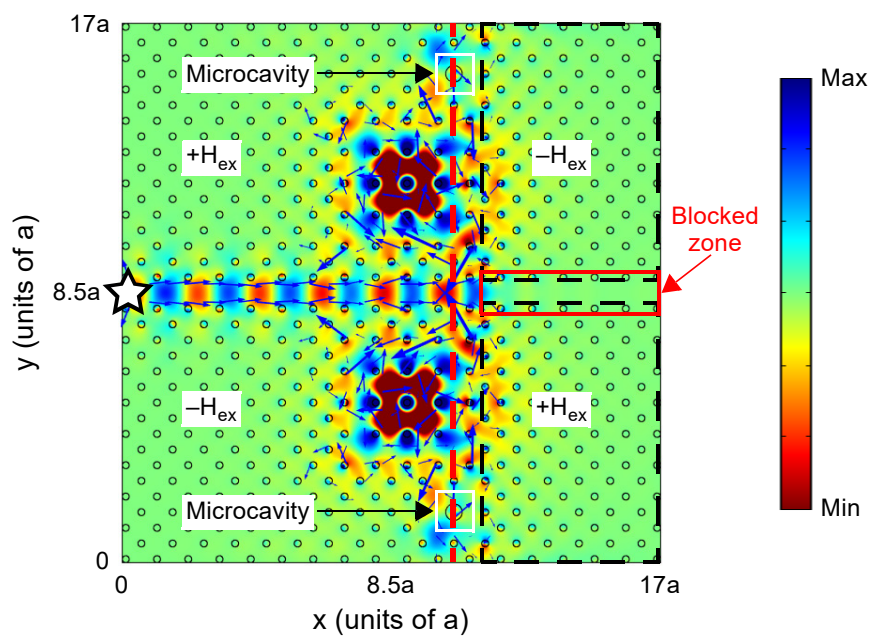

Fig. 9. The field distribution $\left(E_{z}\right)$ of waveguide ring resonators with discharging; the white rectangles denote microcavity in the optical circuit (a). The time-average energy flow along the vertical red dashed line (b). 


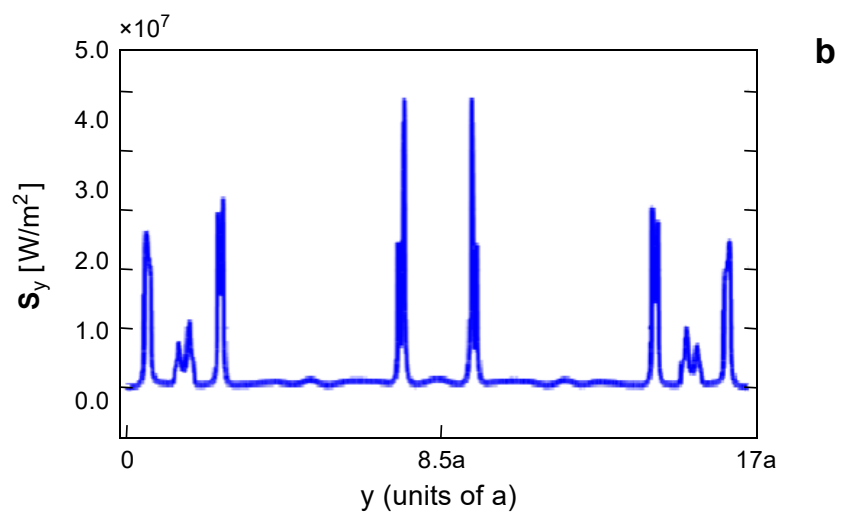

Fig. 9. Continued.

We further perform such a design to realize the storage and discharge of the light information. By increasing the center cylindrical rod radius to $2.2 r$ and removing the nearest four cylindrical rods around the center cylindrical rod, a microcavity is constructed (see Fig. 9a). We can clearly see that the energy of cavity enters the microcavity, namely, photonic energy stored in the cavity flows into the microcavity and circulates along the light wire. Furthermore, we obviously observe the direction of energy flow by the Poynting vector (blue arrows). Here we use a red dashed line crossing two cavities which detect the system time-average energy flow. It is shown that photonic energy of the microcavity is nearly close to energy in the cavities, which means that photonic energy stored by the cavity can be coupled to the microcavity. Then, this configuration realizes the storage and the discharge of photonic energy.

\section{Conclusion}

In conclusion, based on waveguide-cavity interaction, we have proposed a photonic structure composed of two cavities and a unidirectional air waveguide in 2D dielectric PC and a MOPC. The PBGs, dispersion curves of the line defect waveguide and the dispersion curves of waveguide-cavity interaction are calculated and the relations between dispersion curves are also analyzed. Additionally, we have observed waveguide-cavity resonance phenomena with multi-working-channels through numerical simulations with the finite element method. Subsequently, we have proposed an architecture that can dynamically realize storing and discharging of photonic energy. The work we performed offers a more practical pathway for photonic devices and optical circuits at optical frequencies.

Acknowledgments - This work was supported by the National Natural Science Foundation of China (Nos. 61675104 and 91750112), the Natural Science Foundation of Jiangsu Province of China (BK2020), the Sixteenth and Seventeenth Batch of College Students' Scientific Research Project of Jiangsu University (16A164 and 17A172), the Industrial Center's Innovation and Practice Program of Jiangsu University (ZXJG2019102). 


\section{References}

[1] Yablonovitch E., Inhibited spontaneous emission in solid-state physics and electronics, Physical Review Letters 58(20), 1987, pp. 2059-2062, DOI: 10.1103/PhysRevLett.58.2059.

[2] John S., Strong localization of photons in certain disordered dielectric superlattices, Physical Review Letters 58(23), 1987, pp. 2486-2489, DOI: 10.1103/PhysRevLett.58.2486.

[3] Klitzing K.V., Dorda G., Pepper M., New method for high-accuracy determination of the fine-structure constant based on quantized Hall resistance, Physical Review Letters 45(6), 1980, pp. 494-497, DOI: 10.1103/PhysRevLett.45.494.

[4] Laughlin R.B., Anomalous quantum hall effect: an incompressible quantum fluid with fractionally charged excitations, Physical Review Letters 50(18), 1983, pp. 1395-1398, DOI: 10.1103/PhysRev Lett.50.1395.

[5] Raghu S., Haldane F.D.M., Analogs of quantum-Hall-effect edge states in photonic crystals, Physical Review A 78(3), 2008, article 033834, DOI: 10.1103/PhysRevA.78.033834.

[6] Haldane F.D.M., Raghu S., Possible realization of directional optical waveguides in photonic crystals with broken time-reversal symmetry, Physical Review Letters 100(1), 2008, article 013904, DOI: 10.1103/PhysRevLett.100.013904.

[7] Wang Z., Chong Y., Joannopoulos J, Soljačić M., Observation of unidirectional backscattering -immune topological electromagnetic states, Nature 461(7265), 2009, pp. 772-775, DOI: 10.1038/ nature 08293.

[8] Yu Z., Veronis G., Wang Z., Fan S., One-way electromagnetic waveguide formed at the interface between a plasmonic metal under a static magnetic field and a photonic crystal, Physical Review Letters 100(2), 2008, article 023902, DOI: 10.1103/PhysRevLett.100.023902.

[9] Fujita J., Levy M., Osgood Jr. R.M., Wilkens L., Dötsch H., Waveguide optical isolator based on Mach-Zehnder interferometer, Applied Physics Letters 76(16), 2000, pp. 2158-2160, DOI: 10.1063/ 1.126284 .

[10] TAKEDA H., JoHN S., Compact optical one-way waveguide isolators for photonic-band-gap microchips, Physical Review A 78(2), 2008, article 023804, DOI: 10.1103/PhysRevA.78.023804.

[11] Zhang L., Yang D., Chen K., Li T., Xia S., Design of nonreciprocal waveguide devices based on two-dimensional magneto-optical photonic crystals, Optics and Laser Technology 50, 2013, pp. 195 -201, DOI: 10.1016/j.optlastec.2013.03.003.

[12] FU J.-X., LiAN J., LiU R.-J., GAN L., Li Z.-Y., Unidirectional channel-drop filter by one-way gyromagnetic photonic crystal waveguides, Applied Physics Letters 98(21), 2011, article 211104, DOI: $10.1063 / 1.3593027$.

[13] Huang C., Jiang C., Nonreciprocal photonic crystal delay waveguide, Journal of the Optical Society of America B 26(26), 2009, pp. 1954-1958, DOI: 10.1364/JOSAB.26.001954.

[14] Kim S.-H., Ryu H.-Y., Park H.-G., Kim G.-H., Choi Y.-S., Lee Y.-H., Kim J.-S., Two-dimensional photonic crystal hexagonal waveguide ring laser, Applied Physics Letters 81(10), 2002, pp. 2499-2501, DOI: $10.1063 / 1.1510583$.

[15] Dinesh Kumar V., SRinivas T., Selvarajan A., Investigation of ring resonators in photonic crystal circuits, Photonics and Nanostructures-Fundamentals and Applications 2(3), 2004, pp. 199-206, DOI: $10.1016 /$ j.photonics.2004.11.001.

[16] FANG Y., Ni Z., HE H.-Q., JiAng T., Optical storage based on coupling of one-way edge modes and cavity modes, JETP Letters 102(4), 2015, pp. 254-259, DOI: 10.1134/S0021364015160067.

[17] Fang Y., He H.-Q., Hu J., Chen L., Wen Z., Flat and self-trapping photonic bands through coupling of two unidirectional edge modes, Physical Review A 91(3), 2015, article 033827, DOI: 10.1103/ PhysRevA.91.033827.

[18] Fang Y., He H.Q., Hu J., Transforming unidirectional edge waveguide into unidirectional air waveguide, IEEE Journal of Selected Topics in Quantum Electronics 22(2), 2016, pp. 293-301, DOI: 10.1109/JSTQE.2015.2487879. 
[19] FAnG Y., Ni Y., HE H., Hu J., Effect of hybrid state of surface plasmon-polaritons, magnetic defect mode and optical Tamm state on nonreciprocal propagation, Optics Communications 320, 2014, pp. 99-104, DOI: 10.1016/j.optcom.2014.01.043.

[20] Kaliteevski M., Brand S., Abram R.A., Iorsh I., Kavokin A.V., Shelykh I.A., Hybrid states of Tamm plasmons and exciton polaritons, Applied Physics Letters 95(25), 2009, article 251108, DOI: $10.1063 / 1.3266841$.

[21] Wang Z., Shen L., Zhang X., Wang Y., Yu Z., Zheng X., Photonic crystal cavity with one-way rotating state and its coupling with photonic crystal waveguide, Journal of Applied Physics 110(4), 2011, article 043106, DOI: 10.1063/1.3622676.

[22] Liu K., SHen L., He S., One-way edge mode in a gyromagnetic photonic crystal slab, Optics Letters 37(19), 2012, pp. 4110-4112, DOI: 10.1364/OL.37.004110.

[23] Sun Y., Kong X., Fang Y., Competition and transformation of modes of unidirectional air waveguide, Optics Communications 376, 2016, pp. 115-117, DOI: 10.1016/j.optcom.2016.05.038.

[24] Fu J.-X., Liu R.-J., Li Z.-Y., Robust one-way modes in gyromagnetic photonic crystal waveguides with different interfaces, Applied Physics Letters 97(4), 2010, article 041112, DOI: 10.1063/ 1.3470873 .

[25] Wu L.-H., Hu X., Scheme for achieving a topological photonic crystal by using dielectric material, Physical Review Letters 114(22), 2015, article 223901, DOI: 10.1103/PhysRevLett.114.223901.

[26] Yang Y., Xu Y.F., Xu T., Wang H.-X., JiAng J.-H., Hu X., HANG Z.H., Visualization of a unidirectional electromagnetic waveguide using topological photonic crystals made of dielectric materials, Physical Review Letters 120(21), 2018, article 217401, DOI: 10.1103/PhysRevLett.120.217401.

[27] Zhu X., Wang H.-X., Xu C., Lai Y., Jiang J.-H., John S., Topological transitions in continuously deformed photonic crystals, Physical Review B 97(8), 2018, article 085148, DOI: 10.1103/PhysRevB.97.085148.

[28] He L., Gao Y.F., Jiang Z., Wang L.S., Zhou J., Xua X.F., A unidirectional air waveguide basing on coupling of two self-guiding edge modes, Optics and Laser Technology 108, 2018, pp. 265-272, DOI: $10.1016 /$ j.optlastec.2018.06.044.

[29] Gao Y.-F., JiAng Z., Zhang L.-L., He L., Zhao J., Unidirectional propagation of coupled edge states in sandwich topological photonic crystals, Journal of Applied Physics 124(21), 2018, article 213107, DOI: $10.1063 / 1.5047647$.

[30] FAng Y., Ni Z., Xu Q., Zhou J., Wu Y., Transformation of unidirectional modes and split of channel through a cross waveguide, Optics Communications 366, 2016, pp. 13-16, DOI: 10.1016/j.optcom. 2015.12.052. 